\title{
3D Optical Measuring Systems and Laser Technologies for Scientific and Industrial Applications
}

\author{
Yu. Chugui ${ }^{1,2,3,}$, A. Verkhoglyad ${ }^{1}$, A. Poleshchuk ${ }^{4}$, \\ V. Korolkov ${ }^{4}$, E. Sysoev ${ }^{1}$ and P. Zavyalov ${ }^{1}$ \\ ${ }^{1}$ Technological Design Institute of Scientific Instrument Engineering, Siberian Branch of the Russian Academy of Sciences \\ (TDI SIE SB RAS), 41, Russkaya str., Novosibirsk, 630058, Russia, chugui@tdisie.nsc.ru \\ ${ }^{2}$ Novosibirsk State University, 2, Pirogova str., Novosibirsk, 630090, Russia \\ ${ }^{3}$ Novosibirsk State Technical University, 20, K. Marksa pr., Novosibirsk, 630073, Russia \\ ${ }^{4}$ Institute of Automation and Electrometry, Siberian Branch of the Russian Academy of Sciences (IAE SB RAS), 1, \\ Academician Koptyug prosp., Novosibirsk,630090, Russia
}

\begin{abstract}
Modern industry and science require novel 3D optical measuring systems and laser technologies with micro/nanometer resolution for solving actual problems. Such systems, including the 3D dimensional inspection of ceramic parts for electrotechnical industry, laser inspection of wheel pair diagnostic for running trains and 3D superresolution low-coherent micro/nanoprofilometers are presented. The newest results in the field of laser technologies for high-precision synthesis of microstructures by updated image generator using the semiconductor laser are given. The measuring systems and the laser image generator developed and produced by TDI SIE and IAE SB RAS have been tested by customers and used in different branches of industry and science.
\end{abstract}

Keywords: 3D optical inspection, image, shadow, structured light method, low-coherent, interference, laser image generator.

\section{INTRODUCTION}

O OLVING MANY actual safety problems in atomic, space, electrotechnical, and railway branches of industry, as well as in science, requires noncontact 3D optical measurement systems and laser technologies with micro-/ nano-resolution. TDI SIE has researched, developed, implemented and tested some novel optical measuring systems, which are based on the shadow, triangulation, lowcoherent interference methods as well as on the structured light method. Three automatic optoelectronic systems for 3D inspection of different objects in real workshop and outside conditions are described.

The newest results in the field of laser technologies for high-precision recording of microstructures have been obtained by TDI SIE and IAE SB RAS. For these purposes we have developed the updated image generator using a semiconductor laser. High accuracy of element topology synthesis is reached by the precision control systems (resolution on radial coordinate is no worse than $1 \mathrm{~nm}$ ). This generator allows producing the diffractive optical elements (DOE), which are important components of our measurement systems for 3D inspection.

\section{DIMENSIONAL 3D INSPECTION OF CERAMIC PARTS}

Technical ceramic parts are widely used in industry (nuclear, radio electronics, electrotechnical ones, etc.) [1]. A considerable part of them are ceramic parts with diameters from 10 to $50 \mathrm{~mm}$ and thickness from half millimeter to several tens of millimeters (Fig.1.). In many cases these parts have to be produced with high precision (gap tolerance is $10-50 \mu \mathrm{m})$.

Instead of traditional contact methods with known shortcomings (wear of measuring instruments, deformation and even damage of parts, etc.), we have developed an automatic optoelectronic device for 3D noncontact inspection of all geometrical parameters of the ceramic object's family.

The measurement principle of the ring part sizes is illustrated by the optical scheme in Fig.2. For dimensional inspection of these parts we have used three methods: shadow method for measurement of internal and external ring diameters and coaxiality of these diameters, structured light method for measurement of ring height, flatness and parallelism of their face surfaces, as well as TV method for detection of defects on face surfaces.

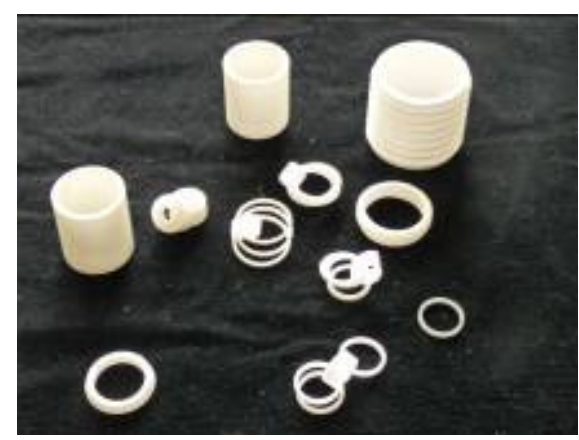

Fig.1. Ceramic parts under 3D dimensional inspection.

The shadow channel consists of LED 1, collimating lens 2, telecentric objective 3 and reception digital camera 5. The inspected part 6 is placed on a table 7 . The structured light channel includes the semiconductor laser 8 , collimating objective 9 and the diffractive optical element (DOE) 10 , forming $N$ light strips. Registration of strips image is carried out by camera 5 through objective 3 . The TV channel includes the ring illuminator 11 , objective 3 and camera 5 . Thus, the same photoreceiver unit is used for all geometrical 
parameter ring inspections by various methods, which provides compactness and simplifies a device design.

The fundamental problem for 3D object inspection by the shadow method is taking into account the influence of thickness $d$ of these parts, and also parameters of the optical system: angular source size $\theta_{s}$ and aperture diaphragm $\theta_{a p}$. In this case the Kirchhoff-Fresnel image formation theory is not valid [2].

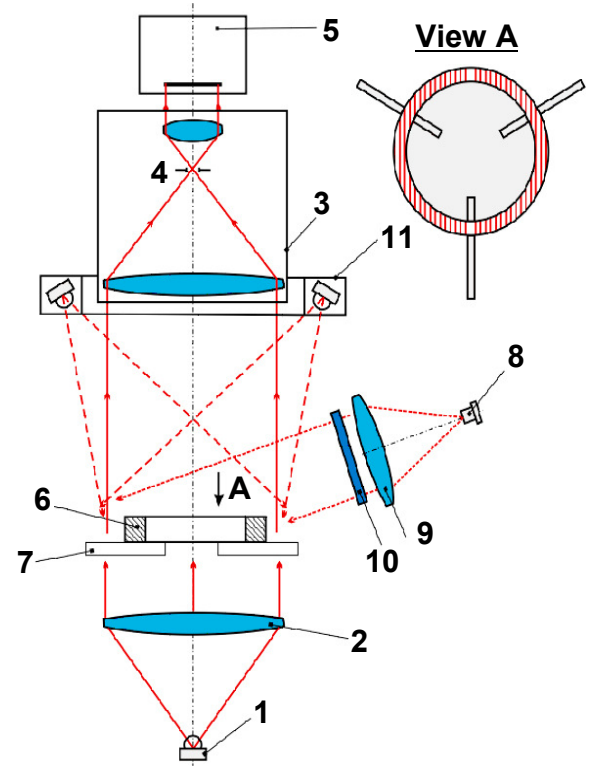

Fig.2. The optical scheme of device for 3D ceramic ring inspection using shadow, structured light and TV methods:

1 - LED, 2 - collimating lens, 3 - telecentric objective,

4 - aperture diaphragm, 5 - digital camera, 6 - inspected part,

7 - table, 8 - semiconductor laser, 9 - collimating objective,

10 - diffractive optical element, 11 - the ring illuminator.

Using the early proposed constructive calculation method for 3D objects we have developed a theory for image formation of ceramic objects in partially coherent light and proposed a threshold algorithm for determination of geometrical positions of boundary objects [3]. It has been shown that the normalized light intensity $I_{t h r}$ in the $3 \mathrm{D}$ (thick) edge image in point $x=0$ (that coincides with object's boundary geometric position) under $\theta_{s}<<\theta_{a p}$ is equal to:

$$
I_{t h r}=I(0)=\frac{1}{4}+\frac{1}{3 \pi^{2}} \frac{\theta_{s}^{2}}{\theta_{a p}^{2}}-\frac{\theta_{a p}}{\sqrt{2} \pi \theta_{c r}},
$$

where $I_{t h r}$ is threshold level, $\theta_{c r}=\sqrt{\lambda / d}$ is critical diffractive angle under which the $3 \mathrm{D}$ effects are significant. Using this model we succeeded to decrease a systematic error of the measuring system from $20 \mu \mathrm{m}$ to $2 \mu \mathrm{m}$.

The structured light method formed by DOE (the ray tracing is shown in Fig.3.) is used for measurement of flatness and parallelism face surface and ring thickness. DOE focuses the laser radiation on ring as 46 light lines with $1 \mathrm{~mm}$ step (Fig.4.). To ensure the equal conditions in the whole measurement range, the incident angle of the rays $\alpha$ and the angular aperture $\Delta \alpha$ for each line have to be identical. The lighting geometry is calculated in such way that the diffraction zero order goes aside from the measuring position. For the correct identification of structured light strips the interval between the 23-rd and 24-th light lines is increased to $1.5 \mathrm{~mm}$ by calculation of DOE structures.

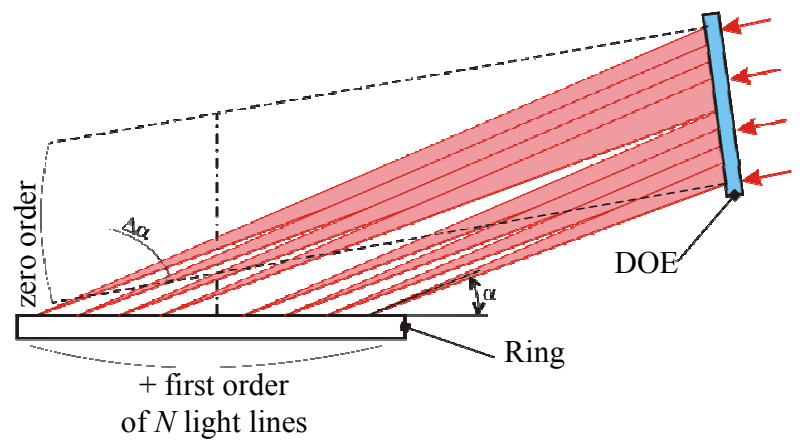

Fig.3. Formation of the structured light using the diffractive optical element for inspection of object's flatness and parallelism.

DOE was produced by laser circular writing system CLWS-300C/M developed at TDI SIE [4, 5]. Due to the binary phase version of DOE its diffractive efficiency is about $40 \%$.

The detection of splits and metallization defects on ceramic ring end faces is a very important problem for customers. For this purpose we used the ring illuminator on the base of 6 light-emitting diodes (located near objective). The illuminator forms the radiation in the field of measuring position with high degree of uniformity. As a rule, the surface defect is located under angle to end face surface. In this case there is light scattering dispersion that leads to formation of the contrast image of defect. Local dispersion characteristics of image intensity, training algorithms and logical rules for decision-making are used for information processing from defective part detection.

a)

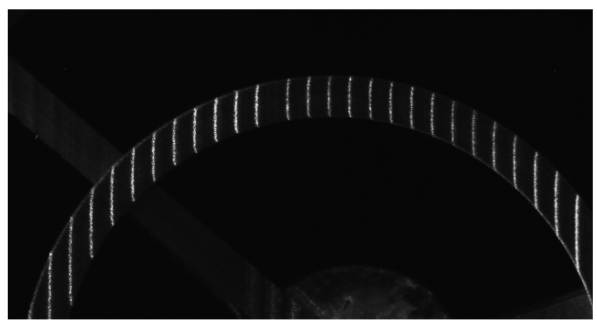

b)

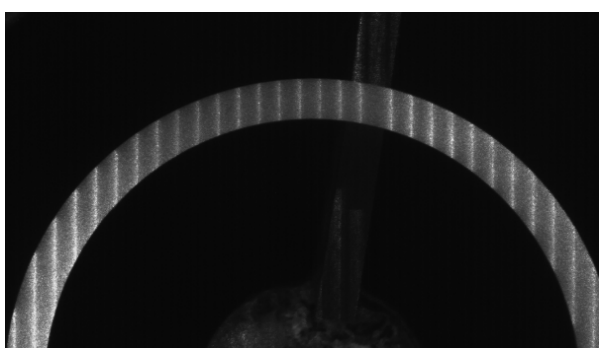

Fig.4. Examples of images of structural lighting strips on the metallized ring (a) and on the ring without metallization (b). 
For JSC "NEVZ" (Novosibirsk, Russia) we have developed and produced an industrial prototype of the optoelectronic system of ceramic ring inspection (Fig.5.).

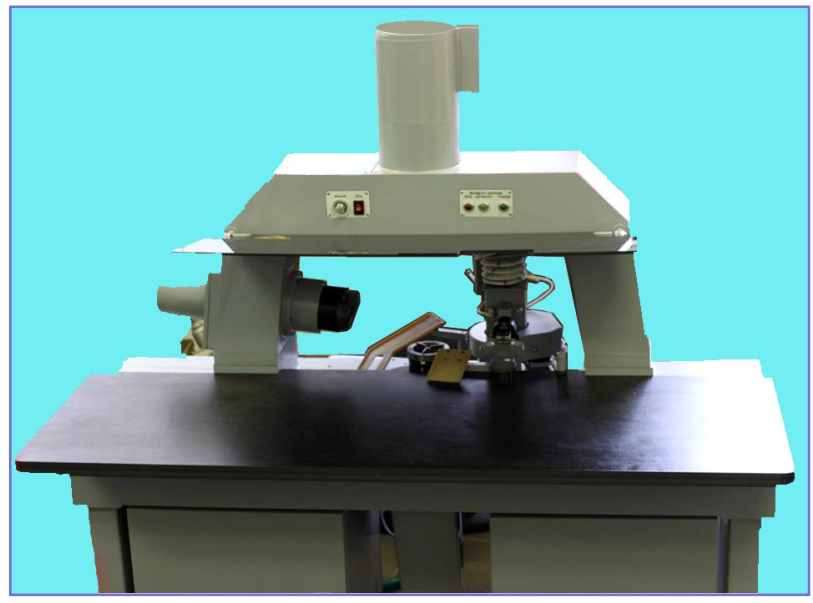

Fig.5. Optoelectronic system RING for 3D ceramic rings inspection.

After the workshop testing of RING system in 2011 the following technical characteristics have been obtained: measuring range of diameters from 20 to $45 \mathrm{~mm}$ and thickness from 0.8 to $10 \mathrm{~mm}$; measurement error of ring geometrical parameters (confidential probability is 0.95 ): external and internal diameters are $\pm 0.006 \mathrm{~mm}$, height is $\pm 0.002 \mathrm{~mm}$, nonflatness of each face is $\pm 0.006 \mathrm{~mm}$ and nonparallelism of two faces is $\pm 0.008 \mathrm{~mm}$. The measurement time is $8 \mathrm{~s}$.

For two years the optoelectronic system RING has been operating successfully at JSC "NEVZ", which is one of the leaders in electrotechnical industry.

\section{LASER WHEEL PAIR DIAGNOSTIC INSPECTION FOR RUNNING TRAINS}

Ensuring the safety of running trains is the actual problem for railways exploitation under passenger and cargo transportation all over the world. Regular inspection of wheels for detection of defects is the main task for train operation safety, especially for high-speed trains. We have investigated the high-speed laser noncontact method (Fig.6., a) for geometrical parameter inspection of moving $3 \mathrm{D}$ objects [6]. Using this method we have developed the automatic all-weather diagnostic system COMPLEX.

The technical solution of the wheel pair parameter inspection problem is based on the principle of self-scanning of wheels using a set of active triangulation type measurement sensors of Labracon ${ }^{\circledR}$ (100 000 meas./s) series where each wheel is scanned in parallel and independently by two (internal and external) measurement sensors. The subsequent combined processing allows determining the rolling surface profile and calculating the required geometrical parameters.

The results of geometrical parameter measurement of running train wheel pairs are stored in the computer database and transmitted to the technical station according to the special protocol.
This protocol includes the time of the train entering and leaving the inspection station, the ordinal number of the wheel pair from the locomotive, types of the wheel pair defect with indication of the defective parameter and its real measured size.

a)

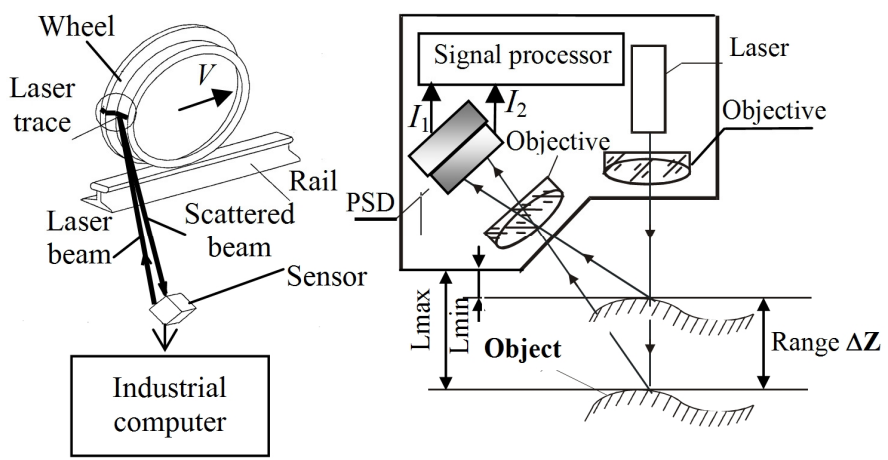

b)
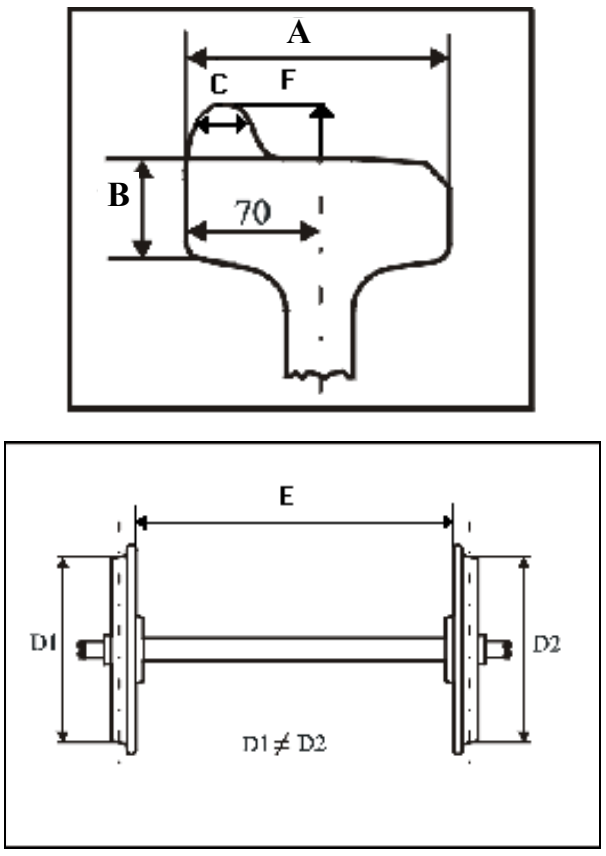

Fig.6. The principle of self-scanning for the running freight car wheels using triangulation measuring sensors (a) and wheel parameters under inspection. (b).

The COMPLEX measures the following parameters of freight car wheel pairs (Fig.6, b):

- rim width $(\mathrm{A})$;

- rim thickness (B);

- flange thickness (C);

- diameter of the rolling surface (D);

- distance between the internal faces of wheels (E);

- uniform rolling $(\mathrm{F})$;

- difference of diameters of wheels in a wheel pair;

- sliding of axle box off the shaft neck. 


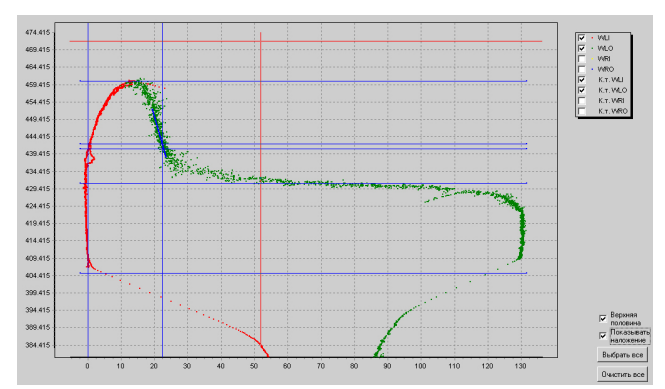

Fig.7. The reconstructed cross-section of the wheel.

The developed algorithms as well as the filtration and signal processing methods allowed to reconstruct the wheel profile with good quality (Fig.7.) and thus to reduce the quantity of axes with incorrectly reconstructed profile under strong natural conditions, such as snow or rain and thereby, to increase reliability of measurement results at speeds of train movement up to $60 \mathrm{~km} / \mathrm{h}$. Measuring error is about $0.5 \mathrm{~mm}$.

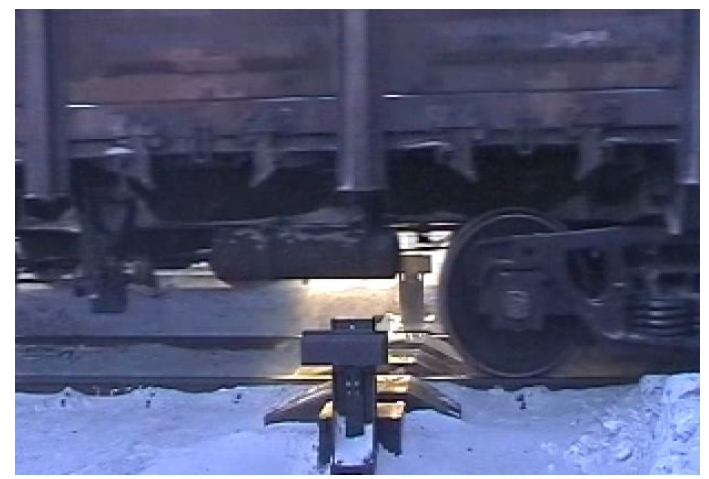

Fig.8. Laser diagnostic system COMPLEX for wheel pair inspection operating in winter conditions at the West-Siberian Railway.

The pilot model of the automatic diagnostic system COMPLEX has been produced and put in continuous operation since 2002 at the West-Siberian Railway (WSR). Using the obtained experience under testing and exploitation of system COMPLEX we have developed its industrial model (Fig.8.). At present, system COMPLEX is an allweather device and is working successfully under the large temperature range from $-50^{\circ} \mathrm{C}$ up to $+50^{\circ} \mathrm{C}$ in conditions of sharply continental climate (similar to Siberian). COMPLEX is unparalleled in the world.

The production of the measuring system COMPLEX, industrial implementation as well as guarantee and postguarantee servicing are carried out by "Siberian Center for Transport Technologies, Ltd." organized by TDI SIE SB RAS together with WSR.

At the present time 80 systems COMPLEX are in operation on 15 Russian regional Railways (from Smolensk to Nakhodka, $9500 \mathrm{~km}$ distance).

By means of the measuring systems COMPLEX the wheel pairs of about five million freight cars are inspected monthly and according to the inspection results about 2500 cars have been uncoupled and sent for the replacement of wheel pairs. The results of such inspection make a great contribution not only to decreasing the accident rates on the railways, but also they allow the Russian Railway Department to predict the wheel pair state using the stored information, as well as to improve methods and technologies of freight car safe operation.

Manufacture of these systems and their installation along the freight train routes will make it possible to draw up short-term and long-term forecasts of behavior of the parameters under inspection. The forecasts will permit establishing the remaining service time for each wheel pair and planning their repair.

Using these systems COMPLEX allows one to improve the safety of railway industry in Russia.

\section{3D SUPERRESOLUTION OPTICAL MICRO/NANOPROFILOMETRY}

Measurement of metallic surface local profile is an urgent problem in many branches of industry, especially for the profile inspection of 3D produced details and their surface defects with micrometer-/ nanometer resolution. TDI SIE has more than twelve years' experience in the development and production of the industrial model of the white-light profilometers.

The principle of object surface profile measurement is based on the interference phenomenon of partially-coherent (in time) light waves, emitted by polychromatic light source (e.g., filament lamp). Measuring device scheme based on Michelson interferometer is shown in Fig.9., a, b [7].

The position and lateral configuration of interference area $(X \times Y)$ on the CCD matrix is determined by intersection of reference surface and surface under measurement. This intersection determines the isoline, corresponding to the given depth along the $Z$ axis in the direction of beam light incident on object. The registration of these areas by a CCD camera allows reconstructing this isoline (Fig.9., b). To obtain a 3D model of the inspected objects, the high precision scanning procedure in depth $Z$ by the change of mutual location of surface under measurement and interferometer unit is used.

We have developed and produced an industrial version of the low-coherent profilometer PROFILE for 3D surface inspection within the range of $0.005-10 \mathrm{~mm}$ with resolution no less than $0.1 \mu \mathrm{m}$ (Fig.10.). These systems have been successfully operating for 10 years in workshop conditions in atomic industry.

In 2010 TDI SIE developed a new type of measuring system - white-light microscope intended both for measurements of micro- and nanoprofile of surfaces by processing of correlograms (Fig.9, c). In this case, under scanning interferometer field within a few nanometers, correlogram is found from the set of interference patterns for each point of the nanosurface [8].

The correlogram maximum corresponding to a zero pathlength difference of light waves scattered by adjacent surface parts in the reference and measuring arms of the interferometer determines the absolute axial position of the measured surface part. The scanning range depends on the coherence length of the light source and can reach tens of microns. 
a)

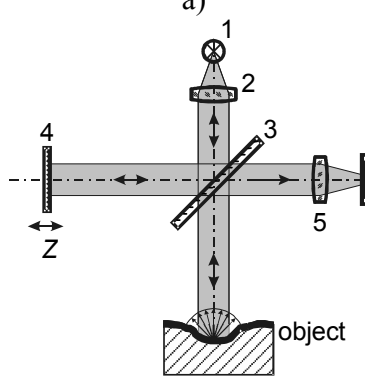

b)

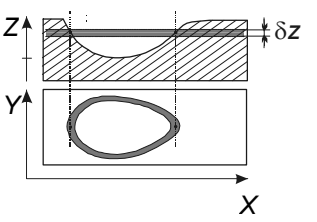

c)

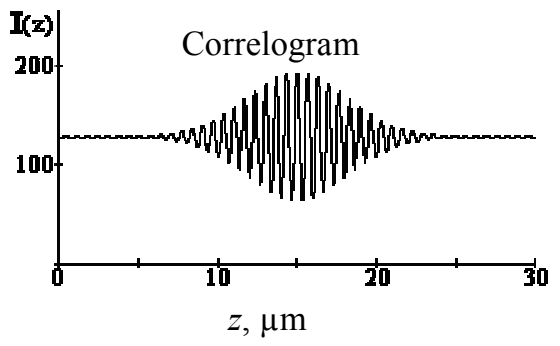

Fig.9. Surface profile measurement principle by white-light interferometry: Michelson interferometer (a), isoline formation (b), correlogram for the coherence length $\approx 15 \mu \mathrm{m}(\mathrm{c}): 1$ - white-light source, 2 - collimator, 3 - split mirror, 4 - reference arm mirror, 5 - objective, 6 - CCD camera, $I(z)$ is the correlogram intensity.

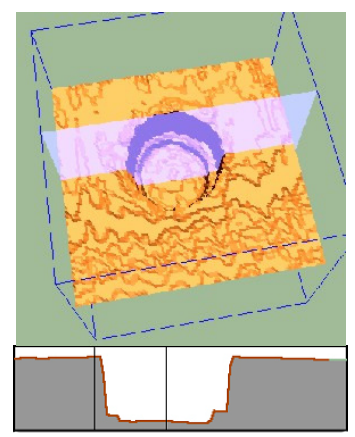

Fig.10. 3D reconstructed closed hole in metal. Area is $0.43 \times$ $0.43 \mathrm{~mm}^{2}$, lateral size is $106 \mu \mathrm{m}$, depth is $115 \mu \mathrm{m}$.

Microscope (Fig.11.) operates in nano- and microregimes. Nanomeasurement regime is intended for measurement of surface relief height of surface finish class with resolution no less than $0.1 \mathrm{~nm}$ up to $50 \mu \mathrm{m}$ (Fig.12.). As for micromeasurement regime the microscope measures "rough" surface relief height with the same specifications as the PROFILE system.

Using a perfect atomic-smooth mirror (produced by Institute of Semiconductor Physics, SB RAS), as well as program-algorithmic software in the interferometer reference arm, the breakthrough under measurement of relief height with resolution of 20 picometers and better has been realized (Fig.13.). 3D-relief of stages (steps) with the height of 1 atom has been reconstructed [9]. The results obtained are the base for metrological task solution in nanometer range, as well as for development of the effective noncontact measuring devices with subatomic in-depth resolution.

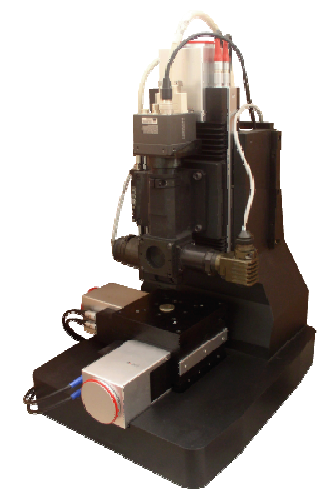

Fig.11. Interference micro/nanoprofilometer MNP-1.

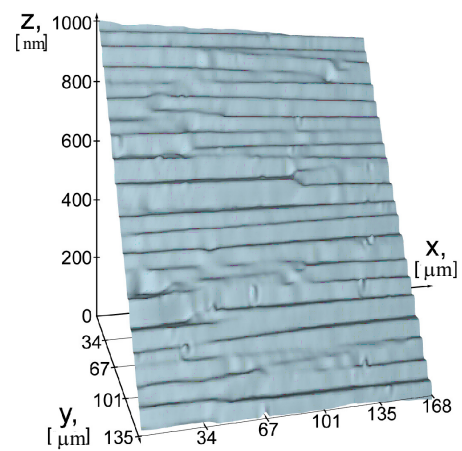

Fig.12. Sample of stepped nanorelief structure on $\mathrm{Si}$ material. Height of atomic step is varied from $5 \mathrm{~nm}$ to $80 \mathrm{~nm}$.

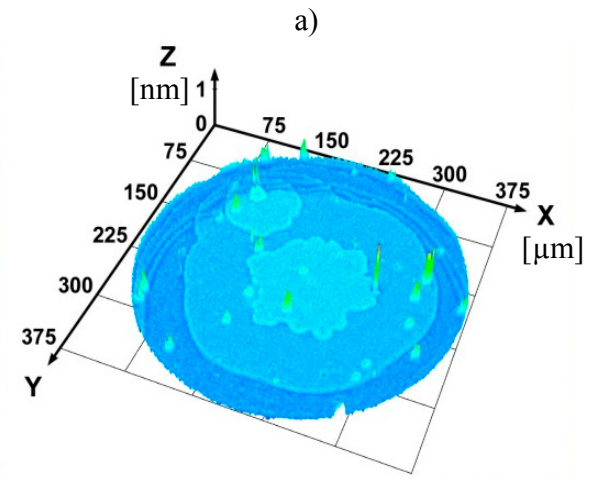

b)

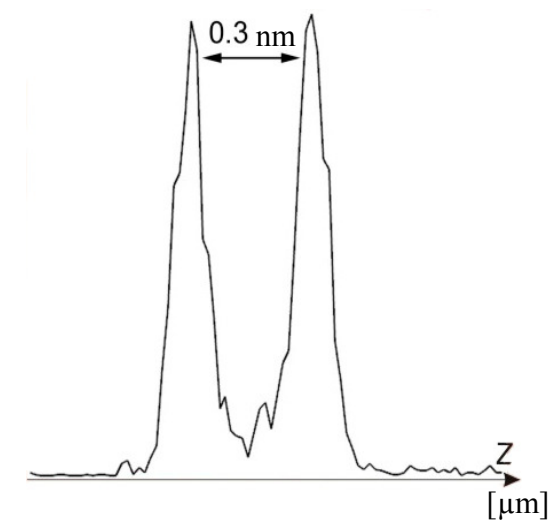

Fig.13. The reconstructed relief of 3D nanoobject (a) and heights histogram (b) under measurement of one-atomic steps on Si crystal surface. 
Application of these micro/nanoprofilometers is very effective for science, including the study of nanostructures and nanomaterials, measurement of film thickness, surface inspection of different polymers, microcrack detection and nanometrology. These profilometers are used also in some institutes of the Siberian Branch of the Russian Academy of Sciences for scientific researches.

\section{UPDATED LASER CIRCULAR IMAGE GENERATOR}

TDI SIE and Institute of Automation and Electrometry SB RAS have jointly developed a new model of circular laser pattern generator using a semiconductor laser for synthesis of high-precision microstructures on flat and curvilinear surfaces.

Laser image generator is working in polar coordinates. The principle of its operation is based on forming the patterns on rotated substrate with photoresist coating by focused laser beam in the mode of circular raster scanning (Fig.14.). The substrate is placed on a high-precision spindle, which is rotated by motor and controlled by spindle module. Laser beam is focused on the substrate by a writing microscope head and its power is changed by acousto-optic modulator under drive control. Radial displacement $R$ is carried out by high-precision air bearing carriage where laser modulator and writing microscope head are placed. This displacement $R$ is measured by laser interferometer. The writing of information on curvilinear surfaces is fulfilled by movable writing microscope head (in vertical $Z$ direction) using autofocus module. High accuracy of element topology synthesis is achieved by the precision control systems.

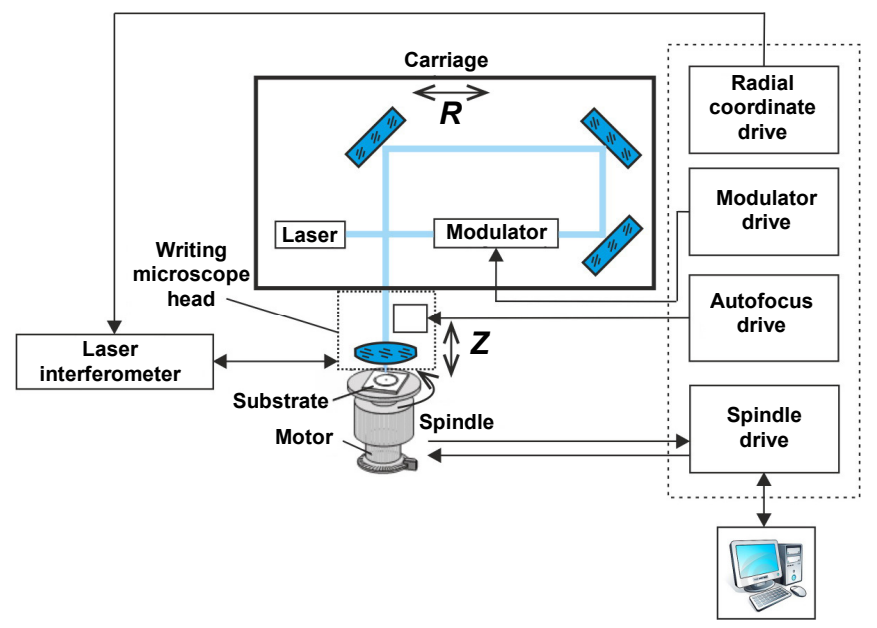

Fig.14. Structure scheme of laser image generator using semiconductor laser.

Placement of current-modulated semiconductor UV laser $(\lambda=405 \mathrm{~nm})$ and optical channel on linear coordinate stage of updated pattern generator allows (as compared with our previous model of laser pattern generator CLWS-300 with argon laser $[4,5]$ ) improving a focused beam pointing stability, to reduce the optical channel length, and to avoid problems connected with water cooling. Multi-channel modulation combining analog and digital direct laser current modulation and acousto-optic modulation broadens a dynamic range of laser beam intensity which is required due to a large range of linear speed at raster scanning.
We have produced a commercial model of laser image generator using a semiconductor laser for the Harbin Institute of Technology (P.R. China) in 2011. As a result of testing of this generator the following main specifications were obtained: working field diameter is $250 \mathrm{~mm}$, spatial resolution is up to $1000 \mathrm{lines} / \mathrm{mm}$, resolution on radial coordinate is no worse than $1 \mathrm{~nm}$, angular coordinate measurement resolution is up to 0.25 ", displacement range on vertical coordinate is up to $25 \mathrm{~mm}$, autofocusing error is $\pm 0.05 \mu \mathrm{m}$. Maximum tilt of curved surface is $10^{\circ}$.

This system allows the formation of diffractive elements, computer-synthesized holograms, gratings, limbs, and different photomasks of arbitrary topology on plane and axially symmetric curvilinear surfaces (Fig.15.).

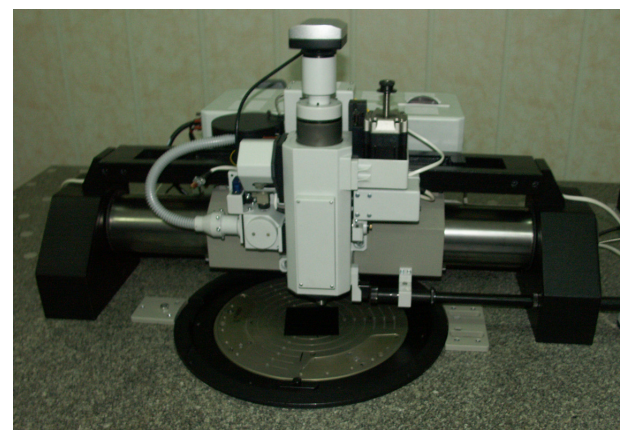

Fig.15. The updated pattern generator using semiconductor UV laser.

This updated laser pattern generator is more compact and has the decreased temperature drift due to special rearrangement of mechanical units. The additional advantages of this generator are the following: periodical rotation center search by means red laser beam with high beam pointing stability, and procedure of interferometer wavelength recalculation during the writing process on the base of environment temperature and air pressure. Controlling software allows one to fabricate binary and continuous relief 3D structures on convex and concave surfaces covered by photoresist or photoresist/Cr layers. The updated system can be easily added by second writing laser with $532 \mathrm{~nm}$ wavelength which is applied to thermochemical direct writing on $\mathrm{Cr}$ films without photoresist. Such process is optimal for high precision computer-synthesized holograms fabricated on large and thick substrates which are not suitable for photoresist spinning.

Example of wavefront distortion map for manufactured binary diffractive lens is depicted in Fig.16. Wavefront error (rms) is about $\lambda / 100$.

\section{CONCLUSION}

The results of the R \& D activity of TDI SIE and IAE SB RAS in the field of 3D optical measuring systems and laser technologies on the base of updated laser image generator are presented.

For electrotechnical industry TDI SIE has developed and produced the optoelectronic device RING for highproductive 3D noncontact inspection of ceramic parts. Using shadow, structured light and TV methods allowed for 8 seconds to perform inspection of all geometrical parameters of ceramic object's family including internal and external 
ring diameters and coaxiality of these diameters, ring height, flatness and parallelism of ring face surfaces, as well as detection of defects on their face surfaces. The system RING has been operating in industry since 2011.

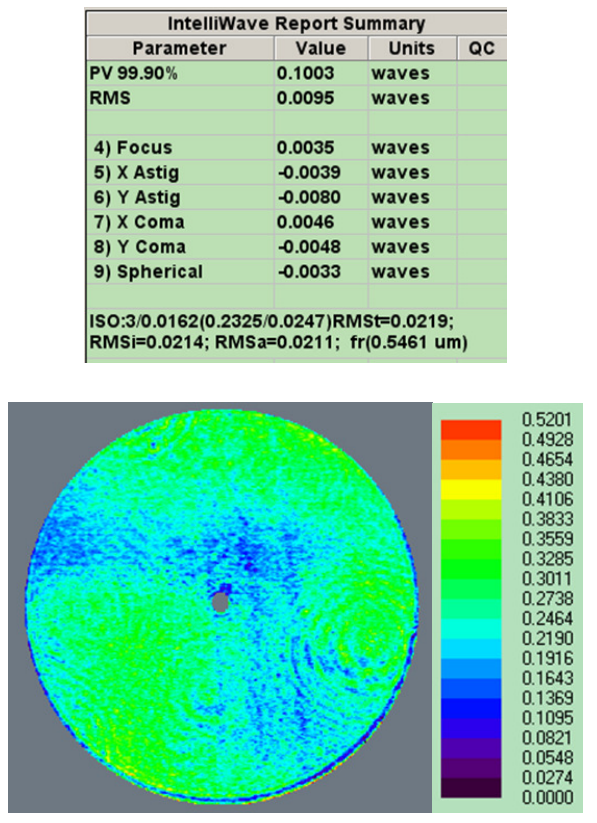

Fig.16. Report summary (from Intellium Z100 interferometer) and wavefront distortion map for the diffractive lens manufactured by the updated laser image generator.

For the first time the automatic all-weather laser diagnostic system COMPLEX for noncontact inspection of geometrical parameters of running freight car wheel pairs was developed by TDI SIE. Measurements are fulfilled at freight car speed up to $60 \mathrm{~km} / \mathrm{h}$ under working temperatures $\pm 50^{\circ} \mathrm{C}$ in conditions of sharply continental climate. At present 80 systems COMPLEX are successfully operating on Russian regional Railways and allow one to improve significantly the safety of railway industry in Russia.

For scientific and industrial applications TDI SIE has developed and produced two models of 3D optical superresolution profilometers using the low-coherent interference method. One of them, the device PROFILE, is intended for 3D microprofiling of different objects with resolution of $0.1 \mu \mathrm{m}$ in wide range up to $10 \mathrm{~mm}$. These profilometers have been in operation during 12 years in atomic industry. The second profilometer model MNP-1 can work in microrange like PROFILE as well as in nanorange with measurement resolution of $0.1 \mathrm{~nm}$ within dynamic range of $50 \mu \mathrm{m}$. Using a perfectly atomic-smooth mirror in the interferometer reference arm, as well as programalgorithmic software, the breakthrough under measurement of relief height with subatomic record resolution of 20 picometers in-depth and better has been achieved. This profilometer can be effectively used in nanoindustry.

For synthesis of high-precision microstructures on flat and curvilinear surfaces TDI SIE and IAE SB RAS have developed and produced a commercial model of the updated circular image generator using a semiconductor laser. High accuracy of element topology synthesis is achieved by the precision control systems. The generator allows one to produce with spatial resolution up to 1000 lines $/ \mathrm{mm}$ within working field diameter $250 \mathrm{~mm}$ on plane and axially symmetric curvilinear surfaces computer-synthesized holograms, gratings, limbs, and different photomasks of arbitrary topology. Effective application of this generator is fabrication of diffractive optical elements for 3D measurement systems. As shown, wavefront error (rms) reconstructed by these elements is about $\lambda / 100$.

The obtained results are applied to many scientific and industrial fields, including atomic, optomechanical, electrotechnical, automobile branches of industry, mechanical engineering, hydropower engineering, space, etc.

\section{REFERENCES}

[1] Technische Keramik. (2004). Brevier technical ceramics. http://www.keramverband.de/brevier_engl /brevier.htm

[2] Born, M., Wolf, E. (2000). Principles of Optics (7th ed.). Pergamon.

[3] Senchenko, E.S., Chugui, Yu.V. (2011). Shadow inspection of 3D objects in partially coherent light. Measurement Science Review, 11 (4), 104-107.

[4] Gurenko, V.M., Kastorsky, L.B., Kiryanov, V.P. et al (2002). Laser writing system CLWS-300/C-M for microstructure synthesis an the axisymmetric 3-D surfaces. In Seventh International Symposium on Laser Metrology Applied to Science, Industry, and Everyday Life. SPIE, Vol. 4900, 320-325.

[5] Chugui, Yu.V., Kiryanov, V.P., Verkhogliad, A.G. (2007). High-performance laser technological processing systems for scientific and industrial applications. In Proceedings of the 35th International MATADOR Conference, 17-20 July 2007. Springer, 139-146.

[6] Baibakov, A.N., Gurenko, V.M., Paterikin, V.I., Yunoshev, S.P., Plotnikov, S.V., Sotnikov, V.V., Chugui, Yu.V. (2004). Automatic control of geometrical parameters of wheel pairs during train operation. Optoelectronics, Instrumentation and Data Processing, 40 (5), 75-82.

[7] Kulikov, R.V., Sysoev, E.V. (2010). Microrelief measurements for a white-light interferometer with adaptive algorithm interferogram processing. Key Engineering Materials : Measurement Technology and Intelligent Instrument IX. Trans Tech Publications, Vol. 437, 35-39.

[8] Sysoev, E.V. (2007). White-light interferometer with partial correlogram scanning. Optoelectronics, Instrumentation and Data Processing, 43 (1), 83-89.

[9] Chugui, Yu.V., Latyshev, A.V., Makarov, S.N., Plotnikov, S.V., Senchenko, E.S., Sysoev, E.V., Verkhogliad, A.G., Zavyalov, P.S. (2011). 3D optical measuring technologies for scientific and industrial applications. In Laser Metrology for Precision Measurement and Inspection in Industry : 10th IMEKO Symposium, 12-13 September 2011. VDI Verlag, Vol. 2156, 13-22.

Received August 26, 2013. Accepted December 11, 2013. 